
EUROPEAN ORGANIZATION FOR NUCLEAR RESEARCH
CERN-SL DIVISION

CERN-SL-2000- 075 (AP)

\title{
Transverse Mode Coupling Instabilities
}

\author{
Jacques Gareyte
}

\begin{abstract}
Transverse Mode Coupling Instabilities (TMCI) emerged between 1974 and 1980 as the main limitation of dense bunches in electron synchrotrons and storage rings. A two-particle model allows one to calculate the Beam Break-Up (BBU) instability in linacs. Extending this to synchrotrons show that the BBU instability is suppressed below a threshold intensity by synchrotron oscillations. The classical theory of head-tail modes together with general properties of coupling impedances are used to show how single bunches become unstable when head-tail modes couple together: this is the TMCI threshold. Above threshold, observations both in proton and electron synchrotron can be described by BBU theory.
\end{abstract}




\title{
Transverse Mode Coupling Instabilities
}

\author{
Jacques Gareyte \\ CERN SL Division, 1211 Geneva 23, Switzerland
}

\begin{abstract}
Transverse Mode Coupling Instabilities (TMCI) emerged between 1974 and 1980 as the main limitation of dense bunches in electron synchrotrons and storage rings. A two-particle model allows one to calculate the Beam Break-Up (BBU) instability in linacs. Extending this to synchrotrons shows that the BBU instability is suppressed below a threshold intensity by synchrotron oscillations. The classical theory of head-tail modes together with general properties of coupling impedances are used to show how single bunches become unstable when head-tail modes couple together: this is the TMCI threshold. Above threshold, observations both in proton and electron synchrotrons can be described by BBU theory.
\end{abstract}

\section{INTRODUCTION}

The concept of mode coupling was invented by F. Sacherer in 1977 to explain the longitudinal turbulence observed on dense particle bunches [1]. However, for reasons which took us almost 20 years to understand, it did not help very much in dealing with longitudinal instabilities, whereas it proved extremely fruitful when applied to transverse collective modes. This report addresses exclusively the problem of transverse mode coupling.

Transverse mode coupling is the ultimate transverse instability, that which remains when all other possible mechanisms have been eliminated. It is observed for single bunches (thereby excluding coupled-bunch instabilities) and for a value of the chromaticity equal to zero (in the absence of head-tail instability).

A first record of what was probably a manifestation of transverse mode coupling appears in 1974: at the $\mathrm{e}^{+} \mathrm{e}^{-}$collider SPEAR I (Standford Linear Accelerator Center) a beating pattern was observed on the coherent betatron signal for large values of the bunch current [2]. A year later SPEAR II was commissioned. This machine had a denser bunch than SPEAR I, and fast losses of the bunch were observed above a threshold current. A vertical instability was suspected, although beam position monitors showed only a weak coherent betatron signal, and the phenomenon persisted for zero chromaticity [3]. This remained a puzzle.

In 1979 the larger $\mathrm{e}^{+} \mathrm{e}^{-}$storage ring PETRA started operation at DESY (Hamburg). Again, fast losses occurred even for zero chromaticity, again a vertical instability was suspected but no large coherent signals were observed. Whereas 


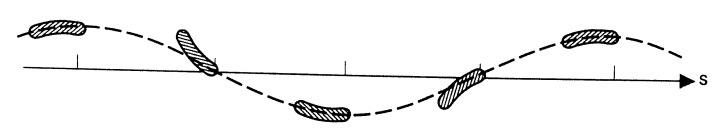

FIGURE 1. Single-bunch beam break-up.
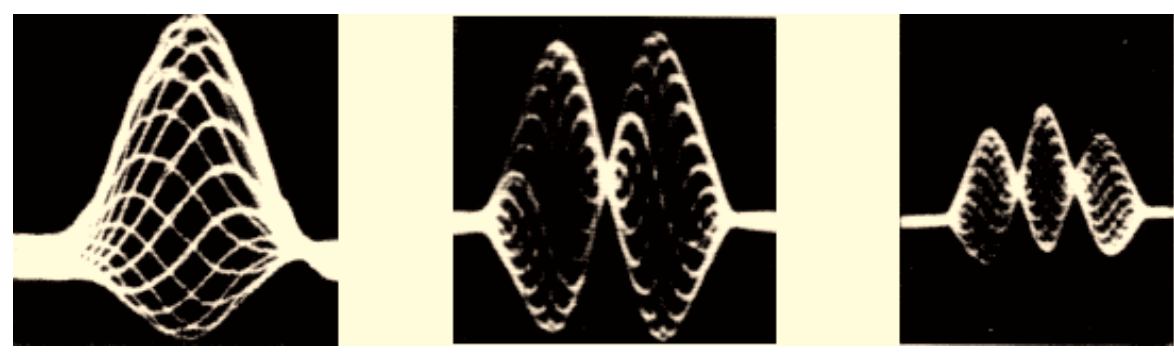

FIGURE 2. Head-tail modes $m=0,1,2$.

at SPEAR II the phenomenon did not really deteriorate the performance of the machine, in PETRA it occurred at a bunch current well below that corresponding to the beam-beam limit, and was therefore a serious limitation.

Although, as already mentioned, F. Sacherer had drawn the attention of the community to mode coupling in 1977, and the extension of this concept to the transverse degrees of freedom was rather straightforward, a suitable theory was not developed immediately. The untimely death of F. Sacherer in a mountain accident in 1978 can probably explain this delay. In 1980 D. Kohaupt proposed the transverse mode coupling mechanism to explain the phenomenon observed in PETRA [4]. It was immediately understood that this was going to be the major limitation of electron storage rings. Kohaupt's paper came just in time to allow a thorough redesign of the injection system of LEP, taking this new phenomenon into account.

In fact, transverse mode coupling is just the manifestation in synchrotrons of a phenomenon which had been known in linacs since the 1960s: the Beam BreakUp instability (BBU) [5]. When a dense bunch of particles travels along a linac, the head of the bunch induces electromagnetic wake fields in the linac structure, which tend to deflect the bunch tail. After a while the bunch tail oscillates about the unperturbed trajectory of the head, as shown in Fig. 1, and can be lost on the aperture. In synchrotrons, the RF longitudinal focusing imposes special patterns of oscillation for the collective transverse motion: the head-tail modes [6]. Examples of head-tail modes are shown in Fig. 2. For low enough bunch intensity, the headtail modes keep their identity, the wake fields merely change their frequency in proportion to the beam current.

Above a certain current, the wake fields are strong enough compared to RF focusing to destroy the head-tail modes: this is the threshold for mode coupling. For currents well above this threshold, the wake fields dominate and the RF focusing can be ignored: we observe the equivalent of the BBU instability in linacs. In 


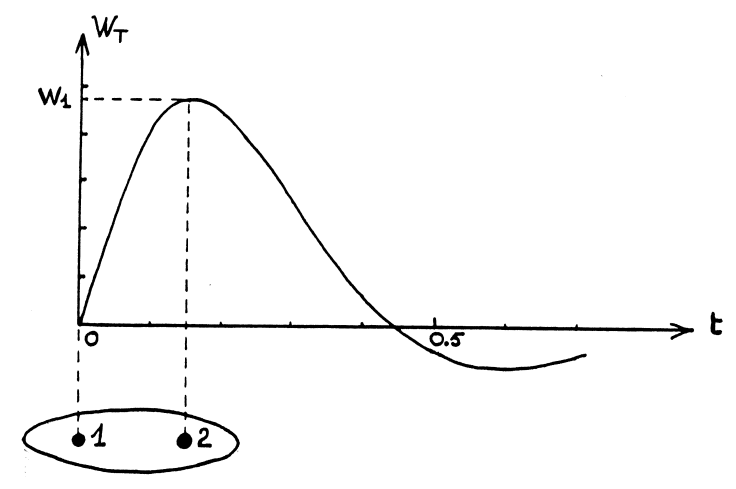

FIGURE 3. Example of transverse wake field $(Q=1$ resonator) generated by head particle 1 , deflecting tail particle 2 .

the following we will first use a simple two-particle model to derive most of the properties of BBU and mode coupling. Then we will outline a general theory of mode coupling based on the standard model of bunched-beam instabilities. Finally we will show that BBU is observed in electron as well as in proton synchrotrons, and that it can be well predicted.

\section{FROM BBU TO MODE COUPLING: A TWO-PARTICLE MODEL}

This subject is treated in detail in Ref. [7]. We start with a model for the wake field. The beam enclosure is made up of a number of elements of various shapes and electrical properties, each one producing its own wake field. We retain only the strong, short-range wake fields, since we are interested in dense, isolated bunches. We assume that the cumulated effect of all the elements can be represented by the response to a shock excitation of a single resonator with a low quality factor $Q$ (we will often take $Q=1$ ), as shown in Fig. 3. This model is very convenient and its validity has been well tested experimentally in many existing machines. As a further simplification, the bunch charge is concentrated in two macroparticles, particle 1 at the head and particle 2 at the tail. Particle 1 excites the wake field, which evolves in time according to

$$
W_{T}=\omega_{r} \frac{R_{T}}{Q} e^{-\epsilon t} \sin S \omega_{r} t
$$

and deflects particle 2 , which in the worst case is situated at the maximum $W_{1}$ of the wake.

In Eq. (1) $\omega_{r}$ is the resonant frequency, $R_{T}$ the maximum resistive component of the corresponding coupling impedance, $S=\left(1-1 / 4 Q^{2}\right)^{1 / 2}$ and $\epsilon=\omega_{r} / 2 Q$. 


\section{The Case of a Linac}

Suppose particle 1 is displaced at time $t=0$ by $y_{1}(0)$. It will oscillate according to

$$
y_{1}=y_{1}(0) \cos \omega_{\beta} t
$$

where $\omega_{\beta}$ is its betatron frequency. The wake force exerted on particle 2 is then (by definition of the wake field) $f=e(N e / 2) y_{1} W_{1}$, so that:

$$
\ddot{y}_{2}+\omega_{\beta}^{2} y_{2}=\alpha y_{1}
$$

with $\alpha=\left(N e^{2} / 2\right)\left(W_{1} / m \gamma\right)$, where $N e$ is the bunch charge, $m$ the particle mass, $\gamma$ the energy devided by $m c^{2}$, and $W_{1}$ the wake field per metre of structure.

The solution of Eq. (3) is:

$$
y_{2}(t)=y_{2}(0) \cos \omega_{\beta} t+y_{1}(0) \frac{\alpha}{2 \omega_{\beta}} t \sin \omega_{\beta} t .
$$

Because particle 2 is driven by particle 1, its amplitude increases linearly with time and at the end of a linac of length $L$ reaches:

$$
\hat{y}_{2}=\hat{y}_{1} \alpha \frac{L}{2 \omega_{\beta} c} .
$$

\section{The Case of a Synchrotron}

\section{General Derivation}

The fundamental difference between a linac and a synchrotron is that in a synchrotron particles 1 and 2 exchange places after half a synchrotron oscillation, $T_{s} / 2$. We can still treat the problem in a simple way if we assume that the wake field is constant between particles 1 and 2 and decays only after the passage of particle 2 . We must furthermore keep track of all possible initial conditions, and we do this by considering the vector

$$
\left|\begin{array}{c}
y_{1} \\
\dot{y}_{1} / \omega_{\beta} \\
y_{2} \\
\dot{y}_{2} / \omega_{\beta}
\end{array}\right| .
$$

The complete solution of Eq. (3) is Eq. (4) with the addition of the terms:

$$
\frac{1}{\omega_{\beta}} \dot{y}_{2}(0) \sin \omega_{\beta} t+\frac{\alpha}{\omega_{\beta}} \dot{y}_{1}(0)\left[\frac{\sin \omega_{\beta} t}{2 \omega_{\beta}^{2}}-t \frac{\cos \omega_{\beta} t}{\omega_{\beta}}\right] \text {. }
$$


We find the solution after half a synchrotron period by putting $t=T_{s} / 2=\pi / \omega s$

$$
\left|\begin{array}{c}
y_{1}\left(T_{s} / 2\right) \\
\frac{1}{\omega_{\beta}} \dot{y}_{1}\left(T_{s} / 2\right) \\
y_{2}\left(T_{s} / 2\right) \\
\frac{1}{\omega_{\beta}} \dot{y}_{2}\left(T_{s} / 2\right)
\end{array}\right|=\left|\begin{array}{cc}
A\left(T_{s} / 2\right) & 0 \\
B\left(T_{s} / 2\right) & A\left(T_{s} / 2\right)
\end{array}\right| \begin{gathered}
y_{1}(0) \\
\frac{1}{\omega_{\beta}} \dot{y}_{1}(0) \\
y_{2}(0) \\
\frac{1}{\omega_{\beta}} \dot{y}_{2}(0)
\end{gathered} \mid
$$

with:

$$
\begin{gathered}
A\left(T_{s} / 2\right)=\left|\begin{array}{cc}
\cos \mu / 2 & \sin \mu / 2 \\
-\sin \mu / 2 & \cos \mu / 2
\end{array}\right| \\
B\left(T_{s} / 2\right)=\Upsilon\left|\begin{array}{lr}
\sin \mu / 2 & \frac{2}{\mu} \sin \mu / 2-\cos \mu / 2 \\
\frac{2}{\mu} \sin \mu / 2+\cos \mu / 2 & \sin \mu / 2
\end{array}\right|
\end{gathered}
$$

where $\mu=2 \pi \omega_{\beta} / \omega_{s}$ and $\Upsilon=\left(\pi N e^{2} W_{1} / 4 m \gamma \omega_{\beta} \omega_{s}\right)$.

By exchanging particle 1 and particle 2 we find the solution for the second half of the synchrotron period, so that the transformation matrix for one complete period is:

$$
T=\left|\begin{array}{ll}
A & B \\
O & A
\end{array}\right|\left|\begin{array}{cc}
A & O \\
B & A
\end{array}\right|=\left|\begin{array}{cc}
A^{2}+B^{2} & B A \\
A B & A^{2}
\end{array}\right|
$$

The characteristic equation of $T$ is complicated. However, knowing that, like in the case of symplectic matrices, the eigenvalues of $T$ can be grouped in reciprocal pairs, we can write it:

$$
\begin{aligned}
(\lambda+ & \left.\frac{1}{\lambda}\right)^{2}+2\left(\lambda+\frac{1}{\lambda}\right)\left[\frac{2 \Upsilon^{2}}{\mu^{2}}(\cos \mu-1)+\Upsilon^{2} \cos \mu-2 \cos \mu\right] \\
& +\left\{4 \Upsilon^{2}(\cos \mu-1)+\left[\Upsilon^{2}+\frac{2 \Upsilon^{2}}{\mu^{2}}(\cos \mu-1)-2 \cos \mu\right]^{2}\right\}=0
\end{aligned}
$$

\section{Simplified Treatment}

The equation for the eigenvalues $\lambda$ can be simplified if we remark that $\mu$ is a large number. For instance in LEP $\omega_{\beta} / \omega_{s}$ is of the order of 1000 . Therefore terms divided by $\mu^{2}$ can be dropped to yield:

$$
\lambda+\frac{1}{\lambda}=-\left(\Upsilon^{2}-2\right) \cos \mu \pm \Upsilon \sin \mu \sqrt{4-\Upsilon^{2}} .
$$

From Eq. (13) it appears that if $\Upsilon>2$, the motion becomes unstable. By writing $\lambda=\exp (i \mu+i \phi)$ it is easy to show that a solution of Eq. (13) is

$$
\sin \phi / 2=\Upsilon / 2
$$

From this we conclude the following: 
- At small intensity $\phi=\Upsilon$. The effect of the interaction is a phase shift.

- As $\Upsilon$ approaches 2, $\phi$ approaches $\pi$. For $\Upsilon>2$, Eq. (14) can only be satisfied with $\phi$ imaginary. Then one of the eigenvalues grows exponentially: this is the Transverse Mode Coupling Instability, or TMCI. Writing $\Upsilon=2+\epsilon$, with $\epsilon \ll 1$, Eq. (14) gives $\cos \phi=1-\Upsilon^{2} / 2=-(1+2 \epsilon)$ and with $\phi=\pi+\Delta \phi$ we obtain $\Delta \phi=2 \sqrt{\epsilon} i$. The amplitude grows like $\exp \left(\Delta \phi t / T_{s}\right)$, that is with a growth rate $1 / \tau=2 \sqrt{\epsilon} / T_{s}$. The square root dependence shows that above threshold but very close to it, the growth rate reaches a value of the order of $1 / T_{s}$.

- Looking at Eq. (5) and the definition of $\Upsilon$, we see that for $\Upsilon=2$, the amplitude of the tail particle grows to twice the amplitude of the leading particle in half a synchrotron period.

We interpret all this in the following way: by periodically exchanging particle 1 and particle 2 the synchrotron oscillation prevents the growth of both particles from accumulating, and therefore stabilizes the beam. However, when the trailing particle grows during half a synchrotron period to twice the amplitude of the leading particle, this stabilizing mechanism ceases to be effective, and the growth does accumulate over many synchrotron periods. For larger values of the synchrotron frequency, the trailing particle has less time to grow before the particles are interchanged, and the threshold of the instability increases.

\section{Synchrobetatron Resonances}

It is instructive to examine the effects which have been neglected by making the approximation $\mu \gg 1$, or $\omega_{\beta} \gg \omega_{s}$. This will introduce us to a phenomenon which is very important in large machines, the coherent synchrobetatron resonance.

We have to solve Eq. (12). The beam will be stable if all solutions for $\lambda+1 / \lambda$ are real and between -2 and 2. Figure 4 shows in grey the unstable areas. We find the well-known instability limit for $\Upsilon>2$, but in addition there are thin areas of instability at smaller beam intensity originating at integer values of $\omega_{\beta} / \omega_{s}$ : these are coherent synchrobetatron resonances. We observe that the width of these additional unstable regions shrinks as $\omega_{\beta} / \omega_{s}$ increases. In the case of LEP where $\omega_{\beta} / \omega_{s} \approx 1000$, one can imagine that they become extremely thin and that our previous approximation is well justified.

However, all the treatment done so far uses a smooth approximation and assumes that the sources of wake fields (the coupling impedances) are smoothly distributed around the machine. This is of course not true, especially in LEP where a large fraction of the coupling impedance is located in the accelerating cavities, which are gathered in a few long straight sections. This introduces azimuthal harmonics of the force. As a consequence, a pattern similar to that of Fig. 4 repeats itself at every integer value of the betatron tune. LEP, with $Q_{s} \simeq 0.1$ and $Q_{\beta} \simeq N+0.25$, is operated in the vicinity of synchrobetatron resonances of order 2 and 3 . It must be 


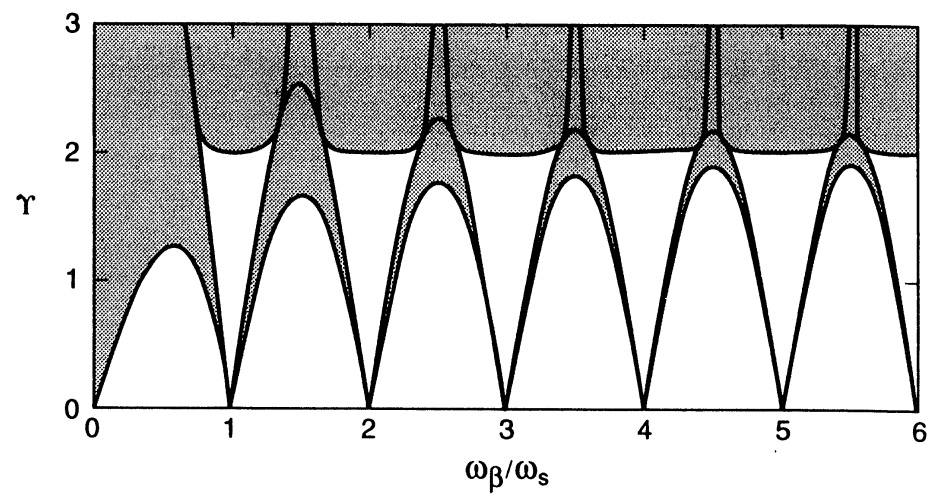

FIGURE 4. Regions of stability for a two-particle beam (shaded is unstable).

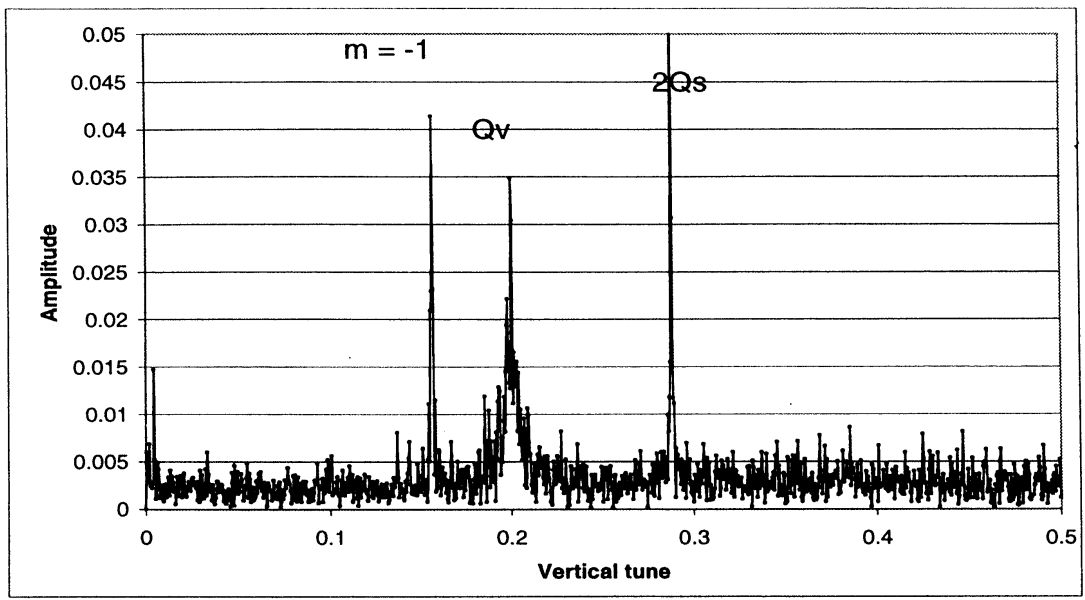

FIGURE 5. Vertical betatron spectrum at LEP, close to TMCI threshold, showing mode $m=-1$ (through its coupling to mode $m=0$ ) and the strong signature of the coherent synchrobetatron resonance $2 Q_{s}$ (here $Q_{s}$ is 0.135$)$.

tuned very carefully during beam accumulation and energy ramping to avoid these resonances. Figure 5 shows a vertical betatron spectrum observed for a bunch current about equal to half the TMCI threshold: the strong line at $2 Q_{s}$ is the signature of the coherent synchrobetatron resonance $Q_{\beta}=N+2 Q_{s}$. If the tune wanders too close to this line, the beam is lost well below the TMCI threshold [8].

\section{HEAD-TAIL MODES AND MODE COUPLING}

A bunch of two particles can oscillate in two different modes: either the particles are in phase (this is mode $m=0$ ) or out of phase (this is mode $m=1$ ). The existence of two modes is just enough to demonstrate the concept of mode coupling. A real bunch exhibits an infinite number of 'head-tail modes'. However, 


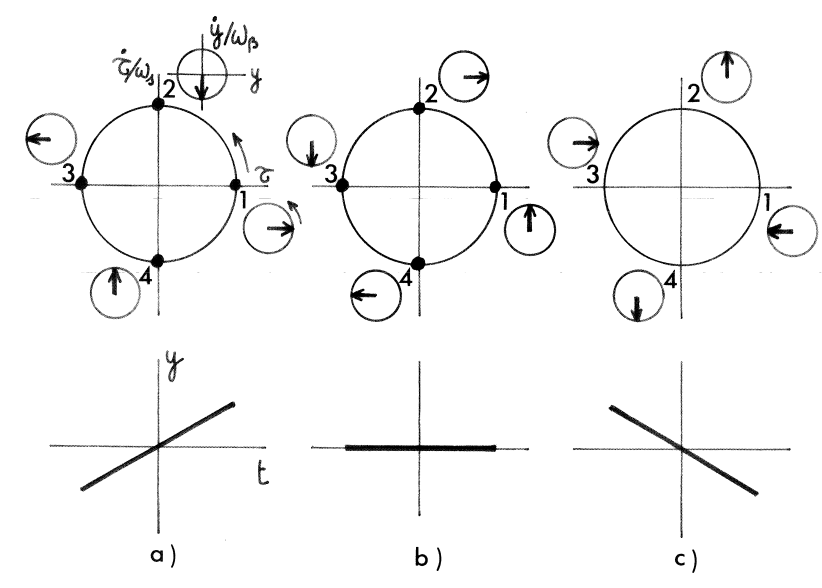

FIGURE 6. Head-tail mode $m=-1$.

the low-order modes $m=0$ and $m=1$ are the easiest to drive and are most often encountered.

In the following we illustrate mode $m=1$ by considering four particles distributed with appropriate betatron phase shift ( $\pi / 2$ between successive particles) along a synchrotron orbit. In Fig. 6 the large circles represent the synchrotron orbit, while the small ones represent the betatron orbits of the four particles. Particle 1 has first maximum positive elongation and particle 3 maximum negative elongation, while particles 2 and 4 are centred. As time proceeds, in the centre picture particles 1 and 3 cross the axis while particles 2 and 4 get maximum but opposite elongation, so that they do not contribute to the coherent signal: the bunch seems to oscillate as a whole around its fixed centre. While this fast betatron motion takes place, the particles showly migrate along the synchrotron orbit, so that after $t=T_{s}$ particle 1 is again at the bunch head. For an external observer, the bunch has made one more oscillation than the individual particles: the frequency of mode $m=1$ is $\omega_{1}=\omega_{\beta}+\omega_{s}$.

With a different initial distribution of betatron phases one can generate mode $m=-1$ with frequency $\omega_{\beta}-\omega_{s}$. In general, mode $m$ has $|m|$ nodes and oscillates at $\omega_{\beta}+m \omega_{s}$.

In our two-particle model it can be shown [7] that the frequency of the two modes 0 and -1 approach each other as the intensity is increased, until they merge for $\Upsilon=2$ and become unstable: hence the name Transverse Mode Coupling Instability (Fig. 7).

A nice illustration of mode coupling is given (Fig. 8) by observations made at the storage ring PEP (SLAC): after the beam has been kicked transversely, one observes a beating pattern with diminishing frequency as modes 0 and -1 approach each other close to TMCI threshold [9]. 


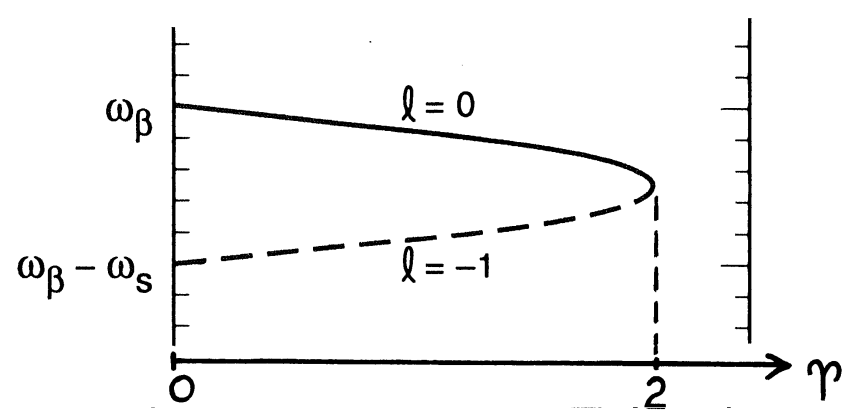

FIGURE 7. Merging of the two coherent modes in the two-particle model.

(a)
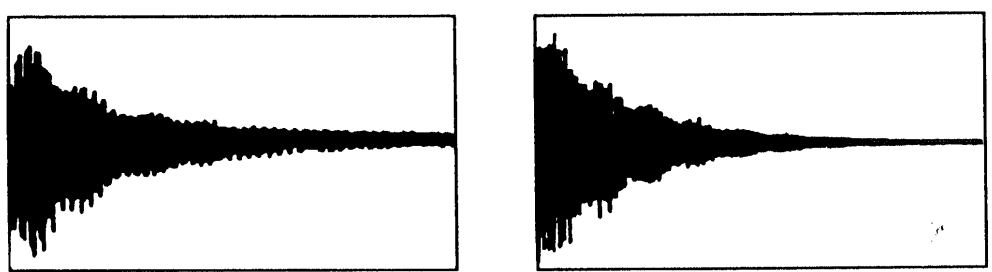

(b)
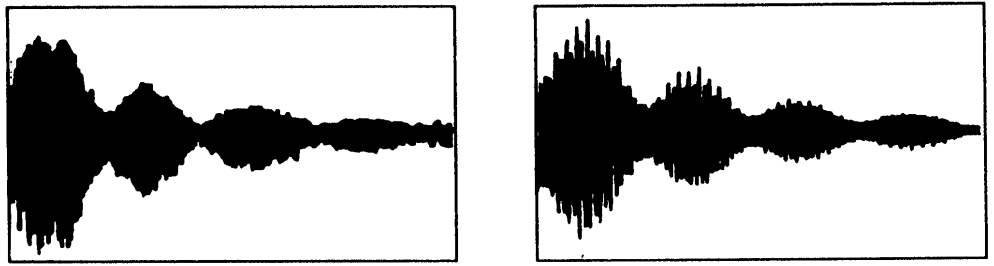

(c)
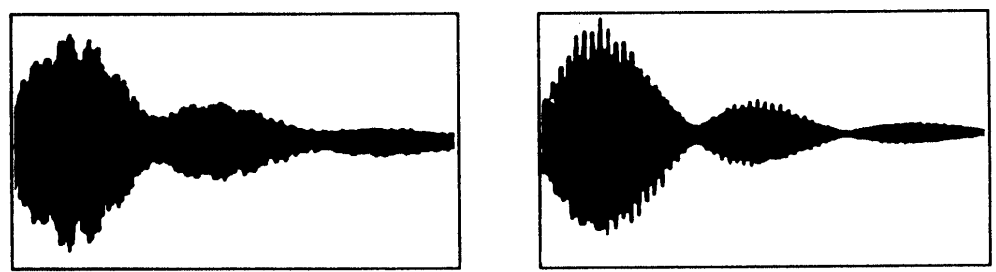

FIGURE 8. Observation (left) and simulation (right) of a beam position monitor signal after the beam has been kicked in PEP. The beam intensity varies between 0.86 (a) and 0.99 (c) of the TMCI threshold. Mode coupling with a decreasing beating frequency is observed. 


\section{OUTLINE OF A GENERAL THEORY OF TMCI}

The principle as well as many fundamental aspects of TMCI can be demonstrated with the two-particle model. To treat the case of a real bunch in a self-consistent way is difficult and beyond the scope of this paper. However, it is relatively easy, using the standard theory of bunched beam stability and invoking basic principles, to demonstrate very interesting and useful properties of mode coupling.

\section{Basics of the Bunched-Beam Stability Theory}

At low intensity, particle bunches in a synchrotron can oscillate transversely according to 'head-tail modes' (see Fig. 2).

A head-tail mode $m$ has $m$ nodes and generates a coherent signal on the synchrotron satellite $m \omega_{s}$ of the betatron lines. Modes are described by a set of orthogonal functions $g_{m}(\omega)$.

In presence of wake fields each mode is coupled to all the others. This is described by the interaction matrix

$$
M_{n}^{m}=C i \sum_{p=-\infty}^{+\infty} i^{m-n} g_{m}(p) Z_{T}(p) g_{n}(p)
$$

where $p=\omega / \omega_{0}$, the frequency divided by the revolution frequency, $Z_{T}$ is the transverse coupling impedance, and $C$ a real constant. At low-intensity modes $m$ and $n$ are separated in frequency by $(m-n) \omega_{s}$ and therefore cannot influence each other significantly. In this situation one can very often neglect the off-diagonal terms in $M$. This is no longer true close to the TMCI threshold when mode frequencies may come close to each other.

Head-tail modes are standing-wave patterns: each point on the mode signal is either in phase or in antiphase with the others. As a consequence their spectra are either symmetric ( $m$ even) or antisymmetric $(m$ odd) with respect to the origin of frequencies, as shown in Fig. 9.

We consider ultrarelativistic beams, in which a particle can only affect those coming behind. Therefore the wake fields obey causality, which means that their Fourier transforms, the coupling impedances, are symmetric (for the reactive part) or antisymmetric (the resistive part) with respect to the origin of frequencies, as shown in Fig. 9 for the $Q=1$ resonator model. Using these general properties and considering formula (15) we see that:

- The effect of a mode $m$ on itself comes only through the reactive part of the transverse coupling impedance $Z_{T}$. It gives a real $M_{m}^{m}$, which means a real frequency shift. There is no possibility of instability if we neglect the off-diagonal terms.

- For two modes $m \neq n$ we have

$$
M_{n}^{m}=(-1)^{m-n} M_{m}^{n} .
$$




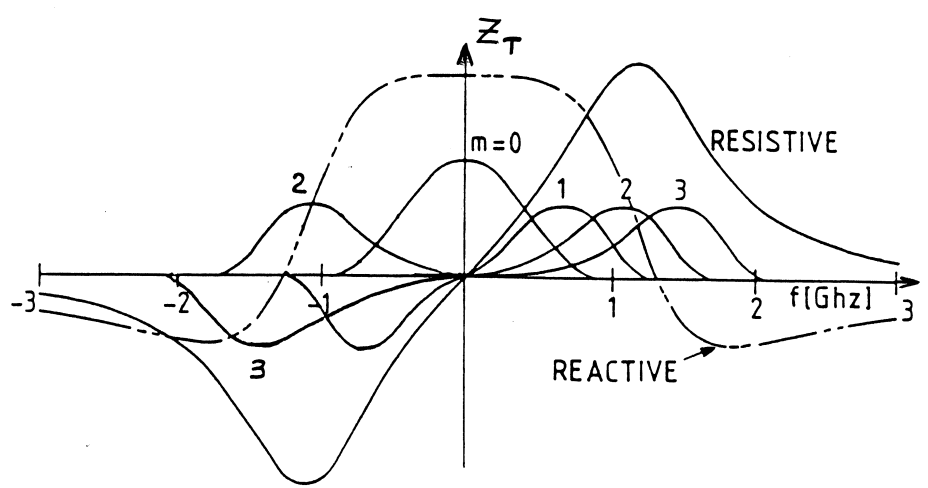

FIGURE 9. Head-tail mode spectra superimposed on the broad-band coupling impedance $(Q=1$ resonator model).

\section{Influence of Mode Parity}

Very often the interaction brings the frequency of two modes close to each other. In this case the dynamics is dominated by the coupling of these two modes. Therefore it is instructive to look at a simplified model in which only two modes interact [10]. Stability is determined in this case by the eigenvalues of a $2 \times 2$ matrix:

$$
\begin{gathered}
\left|\begin{array}{cc}
\lambda-m-M_{m}^{m} & -M_{n}^{m} \\
-M_{m}^{n} & \lambda-n-M_{n}^{n}
\end{array}\right|=0 \\
\lambda=\frac{1}{2}\left(m+M_{m}^{m}+n+M_{n}^{n}\right) \pm \sqrt{\left(m+M_{m}^{m}-n-M_{n}^{n}\right)^{2}+4 M_{n}^{m} M_{m}^{n}} .
\end{gathered}
$$

At low intensity the eigenfrequencies are well separated and the first term under the square root dominates.

In Fig. 10 we describe a situation, which is usual, where this term diminishes as the intensity increases, thus reducing the separation of the eigenmodes. The cross terms then dominate the square root, and two different situations are possible:

- $m$ and $n$ have the same parity. According to Eq. (16) $M_{n}^{m}=M_{m}^{n}$ and the effect of the cross terms is to push apart the eigenmodes. Modes of the same parity repel each other as in Fig. 10(b).

$-m$ and $n$ have different parities. The second term under the square root is negative. As intensity is increased, modes attract each other. For $I=I_{t h}$ they merge and acquire an imaginary component: this is TMCI [Fig. 10(a)].

\section{Influence of Bunch Length}

TMCI is usually observed in electron storage rings, machines which have dense short bunches. Using Fig. 9 it is easy to understand why short and long bunches have very different behaviours. 


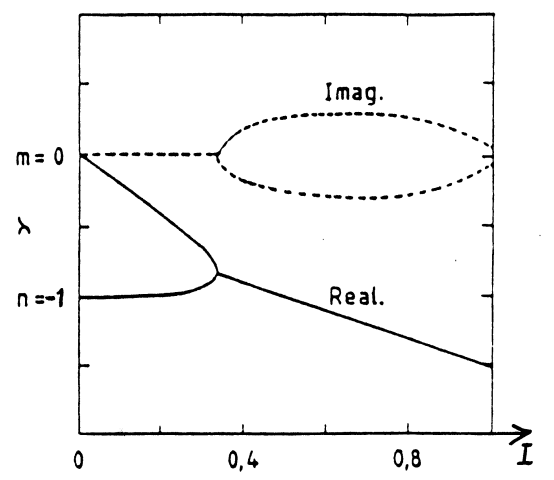

a)

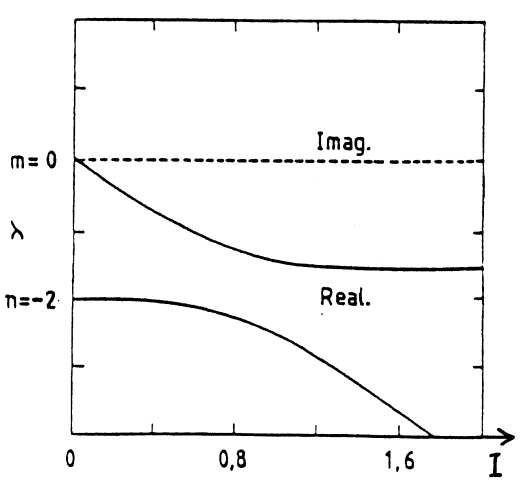

b)

FIGURE 10. Eigenvalues as a function of bunch intensity for the cases $(m-n)$ odd (a) and $(m-n)$ even (b).

A short bunch has a spectrum which extends to high frequency. A typical case is displayed in Fig. 11. Mode $m=0$ interacts strongly with the inductive part of the broad-band coupling impedance and as a result its frequency shifts down as intensity increases. On the contrary, mode $m=-1$ interacts with the capacitive part at higher frequency, and is shifted up towards mode 0 . In addition both modes interact strongly with the resistive part of the coupling impedance, and this results in important cross terms. Therefore all the ingredients are present to generate a strong TMCI. The most unstable case occurs when the bunch length $\sigma_{s}=c / \omega_{r}\left(\omega_{r}\right.$ being the resonant frequency of the broad-band resonator model, or in general the frequency corresponding to the peak of the resistive part of the coupling impedance).

On the contrary, for a long bunch the spectra of modes $m=0$ and $m=-1$ peak at low frequency. Both modes couple to the inductive part of the coupling impedance, and therefore are shifted in the same direction. Moreover, their coupling to the resistive part of the coupling impedance is weak. As a consequence, when the two modes merge, they cannot develop a strong instability and are pulled apart as intensity increases. This behaviour is shown in Fig. 12. Modes of higher order can couple in this case to produce TMCI, but high-order modes are more difficult to drive than low-order ones.

\section{THRESHOLD FORMULA AND CURES}

In the case of short bunches, it is possible to calculate the TMCI threshold in an approximate but very useful way. We neglect the frequency shift of mode $m=-1$ (see Fig. 11) and declare that instability occurs when the tune shift of mode $m=0$ equals $Q_{s}$, the initial tune separation between modes 0 and -1 . We have:

$$
\Delta Q_{(m=0)}=i \frac{I}{2 \pi E / e f_{0}} \frac{1}{2 \pi} \int \beta_{T} Z_{T}(\omega) h\left(\omega, \sigma_{s}\right) d \omega
$$




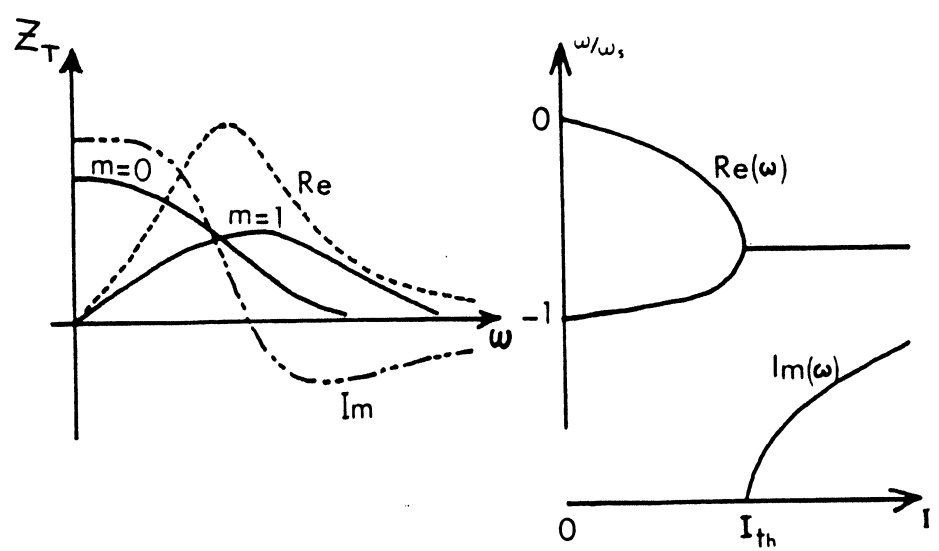

FIGURE 11. Mode coupling for short bunches.
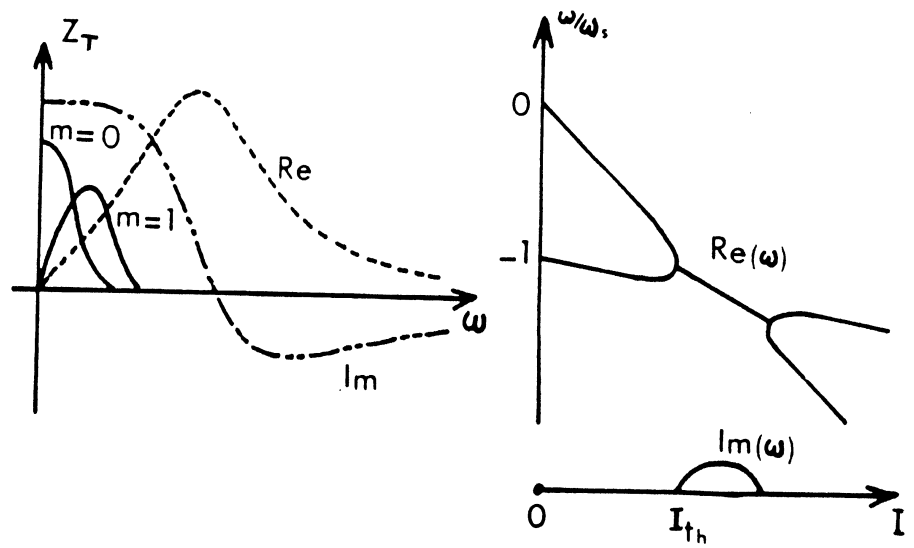

FIGURE 12. Mode coupling for long bunches. 
where $\sigma_{s}$ is the bunch length, $h=g g^{*}$ is the bunch power spectrum, $\beta_{T}$ the betatron function at the location of the coupling impedance $Z_{T}, I$ the bunch current, $E$ the energy and $f_{0}$ the revolution frequency.

Very often the 'kick factor'

$$
k_{T}\left(\sigma_{s}\right)=\frac{1}{2 \pi} \int_{-\infty}^{+\infty} d \omega Z_{T}(\omega) h\left(\omega, \sigma_{s}\right)
$$

is used instead of the impedance. This gives the formula for the threshold current:

$$
I_{t h}=\frac{2 \pi Q_{s} E / e f_{0}}{\sum \beta_{T} k_{T}\left(\sigma_{s}\right)} .
$$

Remark: the threshold found with the two-particle model was:

$$
\Upsilon=2=\frac{\pi N e^{2} W_{1}}{4 m \gamma \omega_{\beta} \omega_{s}} .
$$

Using $\omega_{\beta}=c / \beta_{T}, I=N e f_{0}$, it can be put into the form:

$$
I_{t h}=\frac{16 Q_{s} E / e f_{0}}{\beta_{T}\left(2 \pi R W_{1}\right)} .
$$

For a $Q=1$ resonator model, it can be checked that $\left(2 \pi R W_{1}\right)$, the wake field around the machine, corresponds to $2.6 k_{T}$ for $\omega_{r} \sigma_{s}=1$. Therefore, the two formulae are about the same.

Using Eq. (21) the actions which can be taken to increase the threshold are obvious. In LEP they were all useful and concurred to raise the stored bunch current close to the beam-beam limit. They are:

- increase injection energy from $20 \mathrm{GeV}$ to $22 \mathrm{GeV}$,

- increase $Q_{s}$ up to 0.13 by increasing the RF voltage,

- decrease $\beta_{T}$ at the location of RF cavities,

- decrease $Z_{T}$ by replacing copper cavities by superconducting cavities,

- increase $\sigma_{s}$ by using wigglers.

Another way to counteract TMCI is to use feedback damping, as for any other instability. However, TMCI is a strong instability. As we have seen, just above threshold the growth rate reaches values of the order of $1 / T_{s}$, which for LEP means an $e$-folding time of seven turns. Therefore a very powerful feedback system is required, with all the problems that such a system entails.

Moreover the cross term, which couples two modes, is dominated by the resistive component of the coupling impedance, which peaks at high frequency (see Fig. 9). To be fully effective a feedback should act in the same frequency range; this is very difficult to implement. 


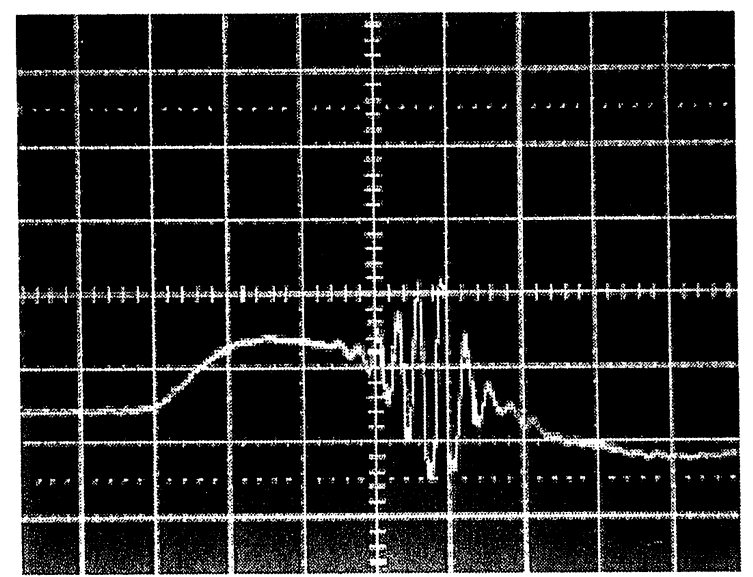

FIGURE 13. Beam break-up on a long proton bunch close to transition in the CERN PS. Horizontal scale: $5 \mathrm{~ns} /$ div.

To go around this problem the use of a 'reactive' feedback was proposed. As we have seen, for short enough bunches the rigid mode $m=0$ is involved. This mode can be influenced at low frequency, and the idea was to counteract its negative tune shift with intensity in an attempt to push the point of crossing with mode $m=-1$ to higher intensity. This worked in PEP (SLAC) and VEPP-4M (Novosibirsk), but for modest values of $Q_{s}$ in the range of 0.02 [11]. Moreover, in PEP at least, it was noted that a usual, resistive feedback had a better performance. A more sophisticated approach was used for LEP [12]. It gave good results in simulation but had a small effect in practice.

\section{ABOVE TMCI THRESHOLD}

We will now illustrate with two examples the dynamics of the instability well above threshold.

For machines with a large value of $Q_{s}$, like LEP, the beam is lost extremely fast and it is difficult to observe anything. In the case of medium or low $Q_{s}$, one can observe the growth of the coherent oscillation.

\section{The Case of Long Bunches: the CERN PS}

Figure 13 show a single-turn signal from a vertical wide-band position monitor [13]. A high-frequency wiggle (at about $700 \mathrm{MHz}$ ) develops towards the tail of the $7 \mathrm{~m}$ long proton bunch. This happens close to transition energy, therefore with a very small $Q_{s}$. In fact, the signal grows much faster than the synchrotron period. The dominating frequency, $700 \mathrm{MHz}$, corresponds to the frequency cut-off of the beam pipe, that is to the peak of the resistive component of the coupling impedance in the classical broad-band model. 
In order to interpret this observation in terms of TMCI theory, one has to invoke mode numbers $m>30$ ! Instead, we propose in this case to use a BBU approach. We consider the synchrotron as a long linac structure of length $L=2 \pi R n$, where $n$ is the number of turns. We neglect synchrotron motion for the time being. We will see later on how we can reintroduce the notion of threshold, even in the absence of $\mathrm{RF}$ voltage.

The theory of BBU developed previously with two macroparticles is not suitable for long bunches. A long train of macroparticles, or bunchlets, is necessary in this case. A suitable theory has been developed for linacs by Yokoya [14] and can be extended to synchrotrons. Using our low- $Q$ resonator model, we calculate the amplitude $y$ of the tail (the last bunchlet) to be after $n$ turns [15]:

$$
y / \delta=\frac{1}{2 \sqrt{2 \pi}} \frac{1}{\omega_{r} \tau} \cdot[\Omega \tau]^{1 / 4} \cdot \exp [-\epsilon \tau+\sqrt{\Omega \tau}]
$$

where

$$
\tau=4 \sigma_{s} / c, \quad \Omega \tau=\frac{N e}{E / e} \beta_{T} \omega_{r} \frac{R_{T}}{Q} \cdot n,
$$

and $\delta$ is the initial amplitude of the bunch. For an instability which develops right after injection into a synchrotron, $\delta$ can be the injection error. When the instability develops at a certain moment in the acceleration cycle, like for instance in the PS close to transition when the synchrotron motion is almost frozen, $\delta$ is the average closed-orbit deviation in the different objects which generate the transverse coupling impedance.

In old machines like the CERN PS or SPS, we have a fair estimate of the coupling impedance after many observations and cross-checks involving different phenomena. Using this estimate and Eq. 23 we can predict the growth rate observed rather well.

\section{The Case of Medium-Length Bunches: the CERN SPS}

We have already considered two extreme cases of bunch length. We have described TMCI in LEP with $\sigma_{s}=1 \mathrm{~cm}$, and BBU in the PS with $\sigma_{s}=1.8 \mathrm{~m}$. Now we will examine the case of the SPS used as a positron and electron accelerator in its role as LEP injector. Bunches are injected in this machine with $\sigma_{s}=16 \mathrm{~cm}$. Irrespective of whether the RF is active or not, for $N$ of the order of $4 \times 10^{10}$ particles we observe the loss of a fraction of the beam after 11 turns, as shown in Fig. 14(b). A wide-band vertical monitor shows a signal growing exponentially from injection up to turn $n=10$, as shown in Fig. 14(a). The signal shown is the output of a broad-band filter centred around $1.5 \mathrm{GHz}$. There is no signal at the bunch frequencies $(<500 \mathrm{MHz})$. Again we observe an instability which grows fast compared to the synchrotron frequency ( $T_{s}$ is 70 turns here), and makes the bunch wiggle at the cut-off frequency of the beam pipe (in the SPS, around $1.3 \mathrm{GHz}$ ).

Applying formula (23) with the well-proven broad-band impedance model of the SPS and $\delta=1 \mathrm{~mm}$, we calculate that the beam tail should be scraped against the beam pipe 15 turns after injection (whereas we observe 11 turns). 


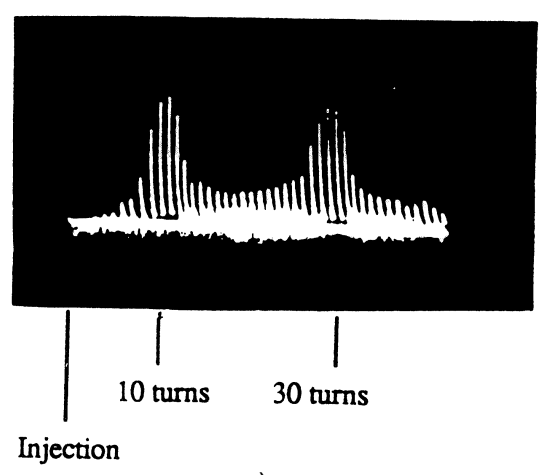

a)

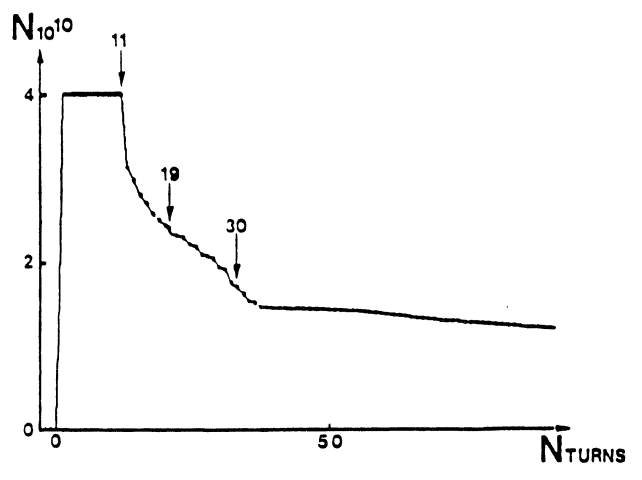

b)

FIGURE 14. Beam position monitor signal filtered around $1.5 \mathrm{GHz}$ (left) and beam intensity (right) as a function of number of turns after injection in the SPS of a positron bunch at $3.5 \mathrm{GeV} / c$.

\section{Threshold of BBU in Synchrotrons}

In the first part of this report we have shown, by using the two-particle model, how the synchrotron motion suppresses the BBU instability below a threshold intensity, by swapping the head and the tail continuously. In fact, in the case of the SPS instability described above, we observe a clear threshold irrespective of whether the RF is active or not. In Fig. $14 N=4 \times 10^{10}$, but for $N \leq 2 \times 10^{10}$ there is no instability.

This can be explained as follows. In a synchrotron, particles of different energies turn at different angular velocities. After a time $T$, particles with a momentum difference $\Delta P$ are separated by a time delay $\Delta T$ given by:

$$
\frac{\Delta T}{T}=\eta \frac{\Delta P}{P}
$$

with $\eta=\frac{1}{\gamma_{t r}^{2}}-\frac{1}{\gamma^{2}}, \gamma_{t r}$ being the $\gamma$ at transition energy.

This differential streaming of particles prevents any build-up of a coherent wave along the bunch if it is too large.

As a rule of thumb let us conjecture that the threshold is reached when the $\Delta T$ accumulated over an $e$-folding time $\tau=T$ is equal to the period of the wave $1 / f_{W}$.

In the SPS we have $\eta=1.85 \times 10^{-3}, \Delta P / P=2 \times 10^{-3}$, and $f_{W}=1.5 \mathrm{GHz}$. The revolution period is $23 \mu \mathrm{s}$. Therefore the instability can be observed only if $\tau<$ 8 turns. This is consistent with observations described above.

\section{CONCLUSIONS}

The beam break-up instability, which is a strong limitation in linacs, is suppressed in synchrotrons below a threshold intensity by the differential streaming of particles in presence of energy spread. In proton accelerators this effect disappears close to transition energy, and BBU can be observed there. 
Below threshold, particle bunches in a synchrotron can oscillate coherently according to well-defined standing-wave patterns, the head-tail modes. For zero chromaticity these modes are stable for single bunches.

However, as the bunch current increases, the wake fields couple head-tail modes together until by merging two modes of different parities, a travelling-wave pattern is created along the bunch. This transition is called Transverse Mode Coupling Instability. Above threshold, the travelling-wave pattern grows in a way very similar to $\mathrm{BBU}$ in linacs.

\section{REFERENCES}

1. Sacherer, F.J., "Bunch Lengthening and Microwave Instability", IEEE Trans. Nucl. Sci. NS-24 (3), 1393 (1977).

2. The SPEAR Group, "Fast Damping of Transverse Coherent Dipole Oscillations in SPEAR", in 9th Int. Conf. on High Energy Accelerators, Stanford, 1974, Conf 740522, AEC, Washington DC, 1974, p. 338.

3. The SPEAR Group, "SPEAR II Performance", in Particle Accelerator Conference, Washington DC, 1975, IEEE Trans. Nucl. Sci. NS-22 (3), 1366 (1975).

4. Kohaupt, R.D., "Transverse Instabilities in PETRA", in 11th Int. Conf. on High Energy Accelerators, CERN, Geneva, 1980, edited by W.S. Newman, Birkhaüser, Basle, 1980, p. 562.

5. Helm, R., and Loew, G., "Beam Break Up", in Linear Accelerators, edited by P.M. Lapostolle and A.L. Septier, North-Holland, Amsterdam, 1970, Chapter B.

6. Gareyte, J., and Sacherer, F., "Head-Tail Type Instabilities in the CERN PS and Booster", in 9th Int. Conf. on High Energy Accelerators, Stanford, 1974, Conf 740522, AEC, Washington DC, 1974, p. 341.

7. Chao, A.W., Physics of Collective Beam Instabilities in High Energy Accelerators, New York, Wiley, 1993.

8. Cornelis, K., "Resonant Behaviour of Head-Tail Modes", in Particle Accelerator Conference, New York, 1999, edited by A. Luccio and W.W. MacKay, Piscataway, IEEE Computer Soc. Press, 1999. Also CERN SL-99-029 OP.

9. The PEP Group, "Comparison Between Experimental and Theoretical Results for the Fast Head-Tail Instability in PEP", in 12th Int. Conf. on High Energy Accelerators, Fermilab, 1983, edited by F.T. Cole and R. Donaldson, Batavia, Fermilab, 1984, p. 209.

10. Chin, Y.H., Transverse Mode Coupling Instabilities in the SPS, CERN/SPS/85-2 (DI-MST).

11. Myers, S., "Stabilization of the Fast Head-Tail Instability by Feedback", in Particle Accelerator Conference, Washington, 1987, IEEE Trans. Nucl. Sci. NS-35, 503.

12. Danilov, V., and Perevedentrev, E., Feedback System for Elimination of the Transverse Mode Coupling Instability, CERN SL/93-38 (AP).

13. Cappi, R., Métral, E., and Métral, G., "Beam Break Up Instability in the CERN PS Near Transition", in 7th European Particle Accelerator Conference, Vienna, 2000. Also CERN/PS 2000-017 (AE).

14. Yokoya, K., Cumulative Beam Break-Up in Large Scale Linacs, DESY 86-084.

15. Brandt, D., Gareyte, J., "Fast Instability of Positron Bunches in the SPS", in 1st European Particle Accelerator Conference, Rome, 1988, World Scientific, Singapore, 1989, p. 690.

16. Gareyte, J., "Observation and Correction of Instabilites in Circular Accelerators", in Frontiers of Particle Beams: Intensity Limitations, Hilton Head Island, South Carolina, 1990, US-CERN School on Particle Accelerators, edited by M. Dienes, M. Month and S. Turner, Lecture Notes in Physics 400, Berlin, Springer, 1992. 